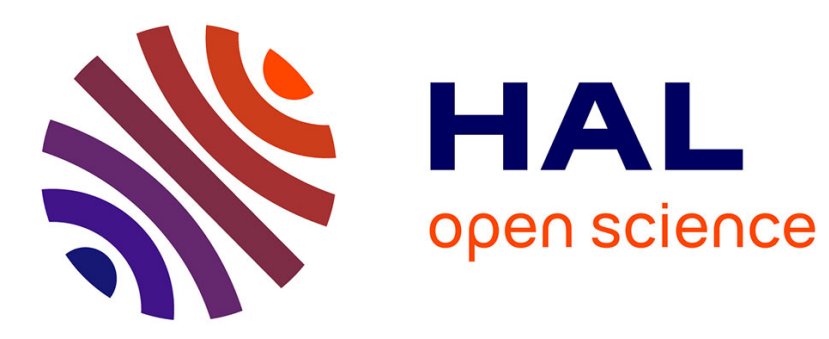

\title{
NMR relaxation in semiflexible Vicsek fractals
}

\author{
Denis A Markelov, Florian Fürstenberg, Maxim Dolgushev
}

\section{To cite this version:}

Denis A Markelov, Florian Fürstenberg, Maxim Dolgushev. NMR relaxation in semiflexible Vicsek fractals. Polymer, 2018, 144 (Mai 2018), pp.65-71. 10.1016/j.polymer.2018.04.022 . hal-02189231

\section{HAL Id: hal-02189231 \\ https://hal.sorbonne-universite.fr/hal-02189231}

Submitted on 19 Jul 2019

HAL is a multi-disciplinary open access archive for the deposit and dissemination of scientific research documents, whether they are published or not. The documents may come from teaching and research institutions in France or abroad, or from public or private research centers.
L'archive ouverte pluridisciplinaire HAL, est destinée au dépôt et à la diffusion de documents scientifiques de niveau recherche, publiés ou non, émanant des établissements d'enseignement et de recherche français ou étrangers, des laboratoires publics ou privés. 


\title{
NMR relaxation in semiflexible Vicsek fractals ${ }^{\text {th }}$
}

\author{
Denis A. Markelova,b, Florian Fürstenberg, ${ }^{\mathrm{a}, 1}$, Maxim Dolgushev ${ }^{\mathrm{d}, *}$ \\ ${ }^{a}$ St. Petersburg State University, 7/9 Universitetskaya nab., St. Petersburg, 199034, Russia \\ ${ }^{b}$ St. Petersburg National Research University of Information Technologies, Mechanics and \\ Optics (ITMO University), Kronverkskiy pr. 49, St. Petersburg, 197101, Russia \\ ${ }^{c}$ Institute of Physics, University of Freiburg, Hermann-Herder-Str. 3, 79104 Freiburg, \\ Germany \\ ${ }^{d}$ Sorbonne Université, CNRS, Laboratoire de Physique Théorique de la Matière Condensée, \\ LPTMC, F-75005 Paris, France
}

\begin{abstract}
We consider the role of local orientational mobility for the NMR relaxation of hyperbranched macromolecules modeled in form of Vicsek fractals. The spectral density $J(\omega)$, that is relevant for the NMR relaxation, is studied for labeled segments of flexible and semiflexible Vicsek fractals. We show that the semiflexibility leads to strong effects. For flexible Vicsek fractals the function $\omega J(\omega)$ has a single maximum, whose position is determined by the local-scale modes and therefore independent of the location of the labeled segments. For the labeled segments of a semiflexible Vicsek fractal, we observe a broad spectral density. It is characterized by the overall relaxation of the branch that originates from the labeled segment and by the overall relaxation of the subbranches that build this branch. In this way, going from the periphery to the inner part of the macromolecule, the labeled segments display a broader spectral density. However, this effect saturates when the labeled segments get closer to the core of the hyperbranched macromolecule, so that they do not feel their remoteness from the periphery anymore.
\end{abstract}

\footnotetext{
In memoriam of Yulii Yakovlevich Gotlib

*Corresponding author

Email address: dolgushev@lptmc.jussieu.fr (Maxim Dolgushev)

${ }^{1}$ Current address: scitech it solutions GmbH, Hermann-Schumann-Str. 4, 16761 Hennigsdorf, Germany
}

Preprint submitted to Polymer

April 4, 2018 


\section{Introduction}

NMR relaxation is one of the most effective experimental methods for investigations of the local mobility of macromolecules [1, 2]. Professor Gotlib, as a pioneer of the theoretical polymer physics, did an immense contribution to the development of the theory of orientanional mobility in various polymeric systems. Starting investigations in this field with polymer chains [3 21], he has also studied more complex systems like polymer networks [22 27] and dendritic macromolecules 28 32]. The significant part of these works has been devoted namely to the role of orientational mobility for the NMR relaxation [6, 7, 1113, 15, 18, 24, 31, 32.

Dendrimers are macromolecules with a specific relaxation spectrum. This feature has led to a quite intricate way in the development of the theory of their NMR relaxation (see, e.g., a recent review [33]). The studies [34 36] devoted to the relaxation spectrum of a dendrimer have put forward the theory of these macromolecules. In the work of Ref. [29] by Gotlib and some of us, the first steps have been done toward the theory of relaxation of local orientation in dendrimers. It has been established that the relaxation of segemental reorientations can be described by two main processes: by the local-scale mobility with an average time of the so-called inner spectrum that is independent of the dendrimer's size and by the overall relaxation (pulsation) of the branch originated from the labeled segment. Thereby it was shown that the corresponding orientational autocorrelation functions depend only on the distance of the labeled segments from the periphery. These theoretical findings have been confirmed by the Brownian dynamics simulations of dendrimers modeled as freely-jointed segments 30, 31, but it was also shown the importance of an additional contribution of the overall rotation of the dendrimer.

The advances in the theory of local orientational mobility in dendrimers allowed for an investigation of spin-lattice relaxation rates $1 / T_{1}$. In Ref. [30] it has been shown that for a dendrimer modeled as a flexible macromolecule the maximum of $\omega / T_{1}$ is determined by the contributions of the local-scale modes 
related to the inner spectrum of the dendrimer. Therefore this maximum does not depend on the location of the labeled segment in the dendrimer as well as on the size of the macromolecule. However, this conclusion was not supported by the experimental studies [37, 38, where the position of the maximum is shifted toward high temperatures (corresponding to low frequencies) for the segments that are closer to the dendrimer's core.

This difference has been overcome by inclusion of the semiflexibility into the classical viscoelastic model based on the framework of Ref. 39. As has been shown in the joint work with Prof. Gotlib 32, introduction of the semiflexibility leads to a shift of the maximum of $\omega / T_{1}$ toward lower frequencies for the segments that are closer to the dendrimer's core. This behavior reflects the fact that inclusion of the semiflexibility leads to a higher contribution of the overall branch relaxation and hence the reorientation of a segment that originates a branch is strongly influenced by the relaxation of that branch. In the following this effect has been confirmed by simulations of semiflexible dendrimers 40 42. Interestingly, the presence of bending rigidity screens the contributions of excluded volume interactions to the NMR relaxation, as has been demonstrated in Ref. 42. Also it has been shown that the hydrodynamic interactions do not change the qualitative picture [43]. Thus, for dendrimers the viscoelastic model extended by inclusion of bending rigidity can fairly describe the local orientational mobility manifested by the NMR relaxation.

The remoteness of the segments from the periphery is also fundamental for other types of macromolecules. In particular this has been observed for the dielectric relaxation of flexible Vicsek fractals [44. The goal of this work is to consider the role of segments' reorientation for the NMR relaxation of fractal hyperbranched macromolecules (modeled in form of Vicsek fractals). For the reasons discussed above the inclusion of semiflexibility is indispensable, and as we proceed to show, it plays a fundamental role. In this work we obtain spectral densities (which determine many NMR relaxation functions) for semiflexible Vicsek fractals and confront them to those of the flexible Vicsek fractals. Also we discuss the differences between NMR relaxation in dendrimers and hyper- 
branched fractals.

The paper is organized as follows. After a brief recall of the architecture of Vicsek fractals, of the main ingredients of the framework of semiflexible treelike polymers, and of the functions related to the relaxation of segmental reorientation, we consider these functions for the segments located in different places in semiflexible Vicsek fractals. The paper is closed by conclusions.

\section{The Model}

\subsection{Vicsek Fractals}

Vicsek fractals 45] (VF) are regular structures that enjoy a growing popularity for the last few decades 44, 46, 75. In Figure 1 we show a VF at generation $G=3$. Moreover, its branching points have functionality (coordination number) $F$ (in Figure 1 it is $F=4$ ). The VF of the next generation is constructed from $F+1 \mathrm{VF}$ of the previous generation. Thus, a VF at generation $G$ consists of $N=(F+1)^{G}$ beads. Changing functionality $F$ allows one to manipulate the scaling properties of the structures. For Gaussian-distributed interbead distances one obtains the $F$-dependent fractal dimension $d_{f}=2 \log (F+1) / \log (3)$ [52. Hence for $F=3$ and $F=4$ the fractal dimension is equal to $d_{f} \approx 2.52$ and $d_{f} \approx 2.93$, respectively, and the VF can be embedded into the three-dimensional space. Note that the first value $\left(d_{f} \approx 2.52\right)$ is very close to that $(2.5)$ found for the quickly grown hyperbranched polymers under the good solvent condition [76] and for the self-avoiding trees under the theta condition [77, 78. The second value $\left(d_{f} \approx 2.93\right)$ is close to the value $d_{f}=3$ found for the hyperbranched trees with annealed connectivity in the melt [79, 80. The regular structure of VF gives a possibility for the calculation of its properties in an iterative way, e.g., the relaxation spectra [52] that is characterized by the spectral dimension [81] $d_{s}=2 \log (F+1) / \log (3 F+3)$. The disordered VF was studied in Ref. [53]; it was found that while the structural irregularities lead to a smoothing of the

relaxation spectrum, the local-scale dynamics reflects the short-range order inherent in the VF. 


\subsection{The framework of semiflexible treelike polymers}

Here we briefly recall the framework of semiflexible treelike polymers (STP), see Ref. 39] for the general approach and Ref. 65] for the particular case of semiflexible VF (SVF).

The main idea of the STP framework lies in the description of a polymer tree through Gaussian-distributed segments $\left\{\mathbf{d}_{i}\right\}$ of the mean-square length $\ell^{2}$, whose orientations are constrained in an average way. In particular, for the adjacent bonds (say $\mathbf{d}_{i}$ and $\mathbf{d}_{j}$ attached to each other by bead $m$ ) one takes $\left\langle\mathbf{d}_{i} \cdot \mathbf{d}_{j}\right\rangle= \pm \ell^{2} q_{m}$, where $q_{m}$ is the stiffness parameter. If $q_{m}=0$, there is no correlation between the segments (so that the junction $m$ is then flexible) and its upper bound depends on the coordination number $F_{m}$ of the $m$ th bead and is given by $1 /\left(F_{m}-1\right)[39,82$. If the segments are connected through the path $\mathbf{d}_{k_{1}}, \ldots, \mathbf{d}_{k_{s}}$, the relation $\left\langle\mathbf{d}_{i} \cdot \mathbf{d}_{j}\right\rangle=\left\langle\mathbf{d}_{i} \cdot \mathbf{d}_{k_{1}}\right\rangle\left\langle\mathbf{d}_{k_{1}} \cdot \mathbf{d}_{k_{2}}\right\rangle \cdots\left\langle\mathbf{d}_{k_{s}} \cdot \mathbf{d}_{j}\right\rangle \ell^{-2 s}$ in spirit of the freely-rotating chain is taken. Thus, we have a full covariance matrix $\boldsymbol{\Sigma}=\left(\left\langle\mathbf{d}_{i} \cdot \mathbf{d}_{j}\right\rangle\right)$ for the Gaussian distribution of $\left\{\mathbf{d}_{i}\right\}$, that leads according to the Boltzmann distribution to the potential energy

$$
V=\frac{3}{2} k_{B} T \sum_{i, j}\left(\boldsymbol{\Sigma}^{-1}\right)_{i j} \mathbf{d}_{i} \cdot \mathbf{d}_{j}=\frac{3 k_{B} T}{2 \ell^{2}} \sum_{m, n} A_{n m} \mathbf{r}_{n} \cdot \mathbf{r}_{m}
$$

In the rhs of Eq. (1) we have introduced the positions $\left\{\mathbf{r}_{m}\right\}$ of the beads that are related to the segments by

$$
\mathbf{d}_{j} \equiv \sum_{k}\left(\mathbf{G}^{T}\right)_{j k} \mathbf{r}_{k}
$$

with the matrix $\mathbf{G}$, which has elements either $G_{n j}=-1$ or $G_{m j}=1$ (if segment $\mathbf{d}_{j}$ points from bead $n$ to $m$ ) and zero otherwise. Moreover, the matrix $\mathbf{A}=$ $\left(A_{n m}\right)$ is related to the matrices $\mathbf{G}$ and $\boldsymbol{\Sigma}$ by $\mathbf{A}=\ell^{2} \mathbf{G} \boldsymbol{\Sigma}^{-1} \mathbf{G}^{T}$. Strikingly, for the treelike polymers A of Eq. (1) has an analytic structure: The diagonal element of $\mathbf{A}$ for a bead of functionality $F$ connected to beads of functionalities $F_{1}, \ldots, F_{F}$ (with corresponding stiffness parameters $q_{F}$ and $q_{F_{s}}$ ) is

$$
\frac{F}{1-(F-1) q_{F}}+\sum_{s=1}^{F} \frac{\left(F_{s}-1\right) q_{F_{s}}^{2}}{1-\left(F_{s}-2\right) q_{F_{s}}-\left(F_{s}-1\right) q_{F_{s}}^{2}} \text {. }
$$


The nearest neighboring beads of functionalities $F_{1}$ and $F_{2}$ lead to

$$
-\frac{1-\left(F_{1}-1\right)\left(F_{2}-1\right) q_{F_{1}} q_{F_{2}}}{\left(1-\left(F_{1}-1\right) q_{F_{1}}\right)\left(1-\left(F_{2}-1\right) q_{F_{2}}\right)}
$$

and two next-nearest neighboring beads connected through a bead of functionality $F$ to

$$
\frac{q_{F}}{1-(F-2) q_{F}-(F-1) q_{F}^{2}} .
$$

All other elements of $\mathbf{A}$ are zeros. The proof of the general structure of $\mathbf{A}$ can be found in Ref. [39]. The analysis of its spectrum for the case of VF is given in Ref. 65].

With the potential of Eq. (1) we can study the dynamics of STP, based on the set of Langevin equations

$$
\zeta \frac{\partial}{\partial t} \mathbf{r}_{k}(t)+\frac{3 k_{B} T}{\ell^{2}} \sum_{n} A_{k n} \mathbf{r}_{n}=\mathbf{g}_{k}(t) .
$$

Here the first term expresses the friction force with the friction constant $\zeta$ and the last term is the zero-mean white-noise force that obeys the fluctuationdissipation theorem, $\left\langle g_{\alpha k}(t) g_{\beta n}\left(t^{\prime}\right)\right\rangle=2 k_{B} T \zeta \delta_{\alpha \beta} \delta_{k n} \delta\left(t-t^{\prime}\right)$ ( $\alpha$ and $\beta$ denote the Cartesian components). Thus, the solution of the set (6) lies in the diagonalization of matrix $\mathbf{A}, \mathbf{Q}^{-1} \mathbf{A Q}=\boldsymbol{\Lambda}$, where $\boldsymbol{\Lambda}$ is the diagonal matrix consisting of the eigenvalues $\left\{\lambda_{i}\right\}$ of $\mathbf{A}$.

\subsection{Relaxation of segments' reorientations}

Based on the solution of Eq. (6) and on the transformation of Eq. (2), we can straightforwardly obtain the bond-bond autocorrelation function $M_{1}^{a}(t)$. The result reads:

$$
M_{1}^{a}(t) \equiv\left\langle\mathbf{d}_{a}(t) \cdot \mathbf{d}_{a}(0)\right\rangle / \ell^{2}=\sum_{\lambda} C_{\lambda}^{a} \exp \left[-t / \tau_{\lambda}\right]
$$

where the sum runs over the distinct nonvanishing eigenvalues $\lambda$ and the corresponding relaxation times are $\tau_{\lambda}=\zeta \ell^{2} /\left(3 k_{B} T \lambda\right)=\tau_{0} / \lambda$. Moreover, the coefficient $C_{\lambda}^{a}$ is given by

$$
C_{\lambda}^{a}=\sum_{k \in \operatorname{ind}_{E V(\lambda)}}\left[\left(\mathbf{G}^{T} \mathbf{Q}\right)_{a k}\right]^{2} / \lambda
$$


where $k$ belongs to the indices of the eigenvector(s) to eigenvalue $\lambda$.

For Gaussian-distributed $\left\{\mathbf{d}_{a}\right\}$, the second Legendre polynomial,

$$
P_{2}^{a}(t) \equiv \frac{1}{2}\left(3\left\langle\frac{\left(\mathbf{d}_{a}(t) \cdot \mathbf{d}_{a}(0)\right)^{2}}{\left|\mathbf{d}_{a}(t)\right|^{2}\left|\mathbf{d}_{a}(0)\right|^{2}}\right\rangle-1\right),
$$

can be expressed analytically based on $M_{1}^{a}(t)$ [83, 84 and written in a compact form 84 ,

$$
P_{2}^{a}(t)=1-3\left\{x^{2}-\frac{\pi}{2} x^{3}\left[1-\frac{2}{\pi} \arctan (x)\right]\right\},
$$

with $x=\sqrt{1-\left(M_{1}^{a}(t)\right)^{2}} / M_{1}^{a}(t)$. The spectral density $J(\omega)$, that is related to the second Legendre polynomial $P_{2}^{a}(t)$ by

$$
J(\omega)=\int P_{2}^{a}(t) e^{-i \omega t} \mathrm{~d} t
$$

is directly involved in the NMR relaxation functions (such as the spin-lattice relaxation time $T_{1}$, see Refs. [1, 2, 85, 86]). We note, however, the situation with the spin-spin relaxation time $T_{2}$ is more complex due to the typically nonexponential dependence of the free induction decay [87].

\section{Results}

\subsection{Segmental autocorrelation function}

The time-dependent autocorrelation functions $M_{1}^{a}(t)$ are calculated using Eq. (7). Here, as in our previous work 44] dedicated to the dielectric relaxation of flexible VF (FVF), we consider the relaxation of reorientation of the core segments belonging to different shells, see Fig. 1. This choice also enables us to make a qualitative comparison of the results for $\mathrm{VF}$ with the behavior of segments in dendrimers 28 32]. In case of semiflexible VF (SVF) the stiffness parameters are $q_{l i n}=0.9$ for the linear junctions and $q_{b r}=q_{l i n} /(F-1)$ for the branching points.

As in the case of dendrimers [29, 32], the functions $M_{1}^{a}(t)$ corresponding to the segments with the same index $m$ (i.e., having the same distance from the periphery), are independent of size $G$ both for FVF and SVF, see Fig. 2 . As for dendrimers [32, it is convenient to analyze this effect by looking at the 
contributions $C_{\lambda}$ (Eq. (8) and Fig. 33) of the relaxation times $\tau_{\lambda}$. The relaxation spectrum of a VF can be distributed in two groups [44, 65]. The first group, the inner spectrum, is related to the local-scale motion and it depends weakly on the macromolecular size. This group of the relaxation times is analogous to the intrachain relaxation spectrum in networks related to the relaxation of a linear spacer inside network's cell [88]. The second group of the spectrum can be interpreted as the pulsation (or overall branch relaxation) spectrum of a dendrimer [34 36]. However, for FVF the pulsating modes are exited not only for the subbranches but also for the inner parts of the macromolecule [44, 52. In case of SVF, the pulsating modes are exited only for the subbranches 65. Therefore the corresponding times $\tau_{m}^{\text {br }}$ have slightly different multiplicities for FVF and SVF [65]. These times have significant contributions $C_{\lambda}^{a}$ to the decay of $M_{1}^{a}(t)$, see Fig. 3

The presence of stiffness leads to a slowing down of the decay of $M_{1}^{a}(t)$ in comparison with FVF (see Fig. 2p. As for dendrimers [32, this is related to the suppression of the small-scale mobility due to the bending rigidity and to the corresponding increase of the contributions related to the slow modes, see Fig. 3. Moreover, the times $\tau_{m}^{\mathrm{br}}$ are much larger for SVF than those for FVF. Also we note an interesting fact, that increasing $m$ leads for SVF to a spreading of the contributions $C_{\lambda}^{a}$ between different $\tau_{m}^{\text {br }}$. While for $m=0$ and $m=1$ there is a dominating relaxation time with the contribution that is larger than 0.5 (i.e., much larger than all other contributions), for $m>2$ the contribution of any relaxation time is not higher than 0.16 . Thus, for high $m$, the width of the part of the spectrum comprising significant relaxation times extends over three to four orders, the finding, which is not shared by the FVF. Hence for SVF there is a quasiplateau of contributions related to the relaxation of branches originating from the segments with indices $m, \ldots, 1$.

Increase of functionality $F$ of the branching units leads to some slowing down of the decay of $M_{1}^{a}(t)$, see Fig. 2. This is related to the fact that a branch with more branched beads has a higher molecular weight, so that its overall relaxation takes a longer time. We note that there are more deviations between 
different $F$ for SVF than for FVF. At the same time the functions $M_{1}^{a}(t)$ for the same $m$ and different $G$ remain to be similar also in case of higher $F$.

\subsection{Spectral density}

In line with the previous works [17 $22,24,32$, we consider the reduced (dimensionless) spectral density

$$
[J(\omega)]=\omega J(\omega)
$$

that is also a convenient representaion for the analysis of experimental data [89].

In case of FVF the position of the maximum of $[J(\omega)]$ does not depend on the parameters of the system; see Fig. 4 for $[J(\omega)]$ of FVF of $G=5, F=3$ and $4, m=0, \ldots, 4$. This feature corresponds to a huge role for FVF of the localscale modes with the relaxation times between $0.2 \tau_{0}$ and $0.6 \tau_{0}$. These times do not depend on the architectural characteristics of VF. The overall branch relaxation (the so-called pulsating modes in case of dendrimers [35]) reveals itself at low frequencies. For segments that are closer to the VF center, the left-hand side (low frequency) wing of $[J(\omega)]$ becomes broader, that is related to increase of contribution of $\left\{\tau_{m}^{\text {br }}\right\}$. Also one can observe an appearance of a non-trivial low-frequency power law, $\omega^{0.8}$ for $F=3$ and $\omega^{0.75}$ for $F=4$. The faster dynamics for lower $F$ is in a qualitative agreement with the dielectric relaxation [44, though the nontrivial mixing of modes (see Eq. 101) deprives us of a simple correspondence of the power laws to the spectral dimension $d_{s}$ of VF.

The function $[J(\omega)]$ for SVF has a much richer behavior than that for FVF. As can be inferred from Fig. $5,[J(\omega)]$ for $m=0$ is characterized by a single maximum, at frequency $\omega \tau_{0} \approx 1.76$ and $\omega \tau_{0} \approx 2.0$ related to the relaxation times $\tau_{0}^{\mathrm{br}}=1.45 \tau_{0}$ and $\tau_{0}^{\mathrm{br}}=1.3 \tau_{0}$ for $F=3$ and $F=4$, respectively. This maximum, as for FVF, is related to the contribution of local-scale modes. For higher $m$, one observes in $[J(\omega)]$ a superposition of several maxima. For $m=1$ there is a maximum at $\omega \tau_{0} \approx 0.06$ and $\omega \tau_{0} \approx 0.047$ related to $\tau_{1}^{\text {br }} \approx 48.5 \tau_{0}$ and $\tau_{1}^{\text {br }} \approx 54.1 \tau_{0}$ for $F=3$ and $F=4$, respectively. For $m=2$ there is a left-hand 
side shoulder at $\omega \tau_{0} \approx 0.003$ and $\omega \tau_{0} \approx 0.0028$ related to the contribution of $\tau_{2}^{\mathrm{br}} \approx 793 \tau_{0}$ and $\tau_{2}^{\mathrm{br}} \approx 987 \tau_{0}$ for $F=3$ and $F=4$, respectively. For higher values of $m$ the curves saturate on each other, showing that the contributions of $\tau_{3}^{\text {br }}$ or $\tau_{4}^{\text {br }}$ are rather low. Also we cannot find a non-trivial power law, as it was observed by FVF.

In this way, the analysis of $[J(\omega)]$ for SVF allows to conclude that the overall branch relaxation times $\tau_{m}^{\text {br }}$ reveal themselves through superimposed maxima leading to a very broad shape of the spectral density. Consequently, the functions for inner segments, $m>2$, overlap practically each other. Thus, these segments do not feel the size of the branch "hanging" on them. This behavior differs from that of dendrimers 32 , where the core segments clearly manifest the size of the "hanging" branch. In Fig. 6 we present a direct comparison of $[J(\omega)]$ for SVF and semiflexible dendrimers (SD) [32, 43] of the same functionality $F=3$, generation $G=5$, and stiffness parameter $q_{\mathrm{br}}=0.45$. As can be inferred from the figure, only the peripheral shell $(m=0)$ shows for both structures nearly the same behavior. All other shells $m \geq 1$ have for SVF and SD significantly different spectral densities. For SD the global maximum of $[J(\omega)]$ is always at lower frequencies for higher $m$; there is no saturation for high $m$ as for SVF. Moreover, the global maxima of $[J(\omega)]$ for SD have a much narrower shape than those for SVF.

\section{Conclusions}

In this study we have investigated the segmental reorientation in fractal macromolecules concentrating on the key function for the NMR relaxation - the spectral density $[J(\omega)]$ of the second Legendre polynomial. A particular focus of our investigations has lain on the role of bending rigidity of the macromolecules.

It has been shown that inclusion of the bending rigidity changes tremendously the behavior of $[J(\omega)]$. In case of flexible fractal macromolecules (studied in form of Vicsek fractals $(\mathrm{VF})$ ), it has a single maximum whose position is independent of the structural parameters $(m, G$ and $F)$. In the low frequency domain for flexible VF appears a region of a nontrivial scaling, with power laws 
$\omega^{0.8}$ for $F=3$ and $\omega^{0.75}$ for $F=4$. This region enlarges for segments that closer to the center of the macromolecule, given that for these segments the large relaxation times are more important. Now, for semiflexible VF $[J(\omega)]$ can be viewed as a single peak only for terminal segments, $m=0$. In all other cases $[J(\omega)]$ has a shape of superimposed peaks. The high-frequency peak (for $m=0$ and as a part of a broad peak for $m>0$ ) is related to the small-scale relaxation, as in the case of flexible VF. The other processes appearing for $m>0$ at low frequencies correspond to overall relaxation of (sub)branches. For the case $m=1,[J(\omega)]$ can be viewed as a superposition of two processes and for $m=2$ of three processes. For $m>2$ the curves saturate on each other, so that the inner segments do not feel anymore their distance to the macromolecule's periphery. Thus, we see that the local orientational behavior of fractal hyperbranched macromolecules differs very much from that of dendrimers, for which the maximum of the reduced spectral density clearly indicates the remoteness of the segments from the dendrimer's periphery [32, 33, 37, 38, 40,43].

\section{Acknowledgments}

D.A.M. acknowledges Government of the Russian Federation (project no. 074-U01) and the Computer Resources Center of Saint Petersburg State University. M.D. acknowledges the support by the European Research Council Starting Grant No. FPTOpt-277998.

[1] R. Kimmich, N. Fatkullin, Polymer chain dynamics and NMR, Springer, 2004.

[2] R. Kimmich, NMR: tomography, diffusometry, relaxometry, Springer Science \& Business Media, 2012.

[3] Y. V. Anufrieva, Y. Y. Gotlib, M. G. Krakovyak, S. S. Skorokhodov, Polarized luminescence study of intramolecular mobility of macromolecules in solution, Polym. Sci. USSR 14 (1972) 1604-1629. 
[4] E. V. Anufrieva, Y. Y. Gotlib, M. G. Krakovyak, I. A. Torchinskii, T. V. Sheveleva, B. V. Shestopalov, Study by polarized luminescence method of molecular-weight dependence of rotational mobility of macromolecules in solution, Vysokomol. Soedin. Ser. A 15 (1973) 2538-2548.

[5] Y. V. Anufrieva, Y. Y. Gotlib, I. A. Torchinskii, Polarized luminescence for longitudinal components of radiation oscillators in the main chain of labelled macromolecules (polymethyl methacrylate), Polym. Sci. USSR 17 (1975) 1343-1352.

[6] Y. Y. Gotlib, M. I. Lifshits, V. A. Shevelev, Proton magnetic relaxation in concentrated polystyrene solutions, Polym. Sci. USSR 17 (1975) 1563-1573.

[7] Y. Y. Gotlib, M. I. Lifshits, V. A. Shevelev, Proton magnetic relaxation in concentrated polymethylmethacrylate solutions, Polym. Sci. USSR 17 (1975) 2132-2142.

[8] I. A. Torchinskii, A. A. Darinskii, Y. Y. Gotlib, Effect of internal friction on the polarized luminescence of solutions of macromolecules with luminescent markers in the main chain, Polym. Sci. USSR 18 (1976) 474-481.

[9] Y. Y. Gotlib, I. A. Torchinskii, Relaxation time spectra and relations of polarization luminescence of macromolecules with luminescent tracers, Polym. Sci. USSR 18 (1976) 3181-3190.

[10] Y. V. Anufrieva, Y. Y. Gotlib, M. G. Krakovyak, V. D. Pautov, Polarized luminescence as used for analyses of high frequency twisting vibrations in macromolecules, Polym. Sci. USSR 18 (1976) 3132-3141.

[11] Y. Y. Gotlib, M. I. Lifshits, V. A. Shevelev, I. S. Lishanskii, I. V. Balanina, Effect of the network of hydrogen bonds on proton magnetic relaxation in solutions of copolymers of acrylic and methacrylic acids with styrene, Polym. Sci. USSR 20 (1978) 467-475. 
[12] Y. Y. Gotlib, Y. Y. Svetlov, I. A. Torchinskii, Hydrodynamic interactions and their effect on local high frequency processes in the polymer chain (polarized luminescence, NMR), Polym. Sci. USSR 21 (1979) 1145-1152.

[13] Y. Y. Gotlib, I. M. Neyelov, I. A. Torchinskii, V. A. Shevelev, Distribution of the correlation times and patterns of $13 \mathrm{C}$ nuclear magnetic relaxation and the Overhauser effect, Polym. Sci. USSR 31 (1989) 1976-1983.

[14] Y. Y. Gotlib, I. M. Neelov, I. A. Torchinski, V. A. Shevelev, Anisotropy of local relaxation properties of macromolecules. polarized luminescence, Macromol. Theory Simul. 2 (1993) 1-11.

[15] Y. Y. Gotlib, I. A. Torchinski, V. A. Shevelev, Anisotropy of local relaxation properties of macromolecules. spin-lattice relaxation of $13 \mathrm{C}$ nuclei, the nuclear Overhauser effect and the estimation of parameters of an equivalent ellipsoid for kinetic segments of polymer chains, Macromol. Theory Simul. 2 (1993) 13-20.

[16] Y. Gotlib, Model theories of the interaction between structural and local orientational relaxation processes in polymeric systems, J. Non-Cryst. Sol. 172 (1994) 855-856.

[17] Y. Y. Gotlib, I. A. Torchinskii, V. A. Shevelev, Interplay between internal friction and local macromolecular dynamics, Polym. Sci. Ser. A 37 (1995) 915-920.

[18] Y. Y. Gotlib, I. A. Torchinskii, V. A. Shevelev, Spin-lattice proton relaxation and the dynamics of polymer chains in the gels of cross-linked polystyrene, Polym. Sci. Ser. A 38 (1996) 1287-1292.

[19] Y. Y. Gotlib, I. A. Torchinskii, V. A. Shevelev, Nuclear magnetic relaxation in cross-linked poly (fluoroalkyl acrylates). local orientational order in side chains, Polym. Sci. Ser. A 39 (1997) 1328-1332. 
[20] Y. Y. Gotlib, I. A. Torchinskii, V. A. Shevelev, Spin-lattice relaxation and the nuclear overhauser effect in macromolecules with finite thermodynamic rigidity, Polym. Sci. Ser. A 43 (2001) 1066-1073.

[21] Y. Y. Gotlib, I. A. Torchinskii, V. A. Shevelev, Spin-lattice relaxation and nuclear Overhauser effect in macromolecules with finite thermodynamic rigidity: Comparison of the longitudinal and transverse correlation time spectra, Polym. Sci. Ser. A 44 (2002) 206-214.

[22] V. A. Shevelev, I. A. Torchinskii, Y. Y. Gotlib, Cooperative dynamics of macromolecules in polymer networks and spin-lattice relaxation, Vysokomol. Soedin. Ser. A 41 (1999) 1607-1612.

[23] Y. Y. Gotlib, M. I. Lifshits, V. A. Shevelev, I. S. Lishanskii, I. V. Balanina, The influence of the chemical crosslinking network on the spin-spin relaxation of crosslinked and swelling polymer systems, Polym. Sci. USSR 18 (1976) 2630-2636.

[24] Y. Y. Gotlib, I. A. Torchinskii, V. P. Toshchevikov, V. A. Shevelev, Theory of the relaxation spectra of polymer networks with included hard rodlike particles manifested in NMR, Appl. Magn. Reson. 30 (2006) 657-671.

[25] Y. Y. Gotlib, A. A. Lezova, I. A. Torchinskii, Rotational and translational relaxation times of quasi-elastic and rigid dumbbells elastically bound to polymer network junctions, Polym. Sci. Ser. A 48 (2006) 498-508.

[26] V. P. Toshchevikov, Y. Y. Gotlib, Theory of relaxation spectra and dielectric relaxation of rigid rodlike particles incorporated in a polymer network, Polym. Sci. Ser. A 48 (2006) 649-663.

[27] Y. Y. Gotlib, I. A. Torchinskii, V. P. Toshchevikov, V. A. Shevelev, The viscoelastic coarse-grained dynamic model of the polymer network with embedded rod-like particles. relaxation spectra and mobility of different scales., Macromol. Symp. 252 (2007) 130-139. 
[28] Y. Y. Gotlib, D. A. Markelov, Permittivity of a dendrimer containing polar groups, Polym. Sci. Ser. A 46 (8) (2004) 815-832.

[29] Y. Y. Gotlib, D. A. Markelov, Theory of orientational relaxation of individual specified units in a dendrimer, Polym. Sci. Ser. A 49 (2007) 1137-1154.

[30] D. A. Markelov, Y. Y. Gotlib, A. A. Darinskii, A. V. Lyulin, S. V. Lyulin, Local orientational mobility in dendrimers. theory and computer-aided simulation, Polym. Sci. Ser. A 51 (2009) 331-339.

[31] D. A. Markelov, S. V. Lyulin, Y. Y. Gotlib, A. V. Lyulin, V. V. Matveev, E. Lahderanta, A. A. Darinskii, Orientational mobility and relaxation spectra of dendrimers: Theory and computer simulation, J. Chem. Phys. 130 (2009) 044907.

[32] D. A. Markelov, M. Dolgushev, Y. Y. Gotlib, A. Blumen, NMR relaxation of the orientation of single segments in semiflexible dendrimers, J. Chem. Phys. 140 (2014) 244904.

[33] D. A. Markelov, M. Dolgushev, E. Lähderanta, NMR relaxation in dendrimers, Annu Rep. NMR Spectrosc. 91 (2017) 1-66.

[34] C. Cai, Z. Y. Chen, Rouse dynamics of a dendrimer model in the $\theta$ condition, Macromolecules 30 (1997) 5104-5117.

[35] Y. Y. Gotlib, D. A. Markelov, Theory of the relaxation spectrum of a dendrimer macromolecule, Polym. Sci. Ser. A 44 (2002) 1341-1350.

[36] A. A. Gurtovenko, D. A. Markelov, Y. Y. Gotlib, A. Blumen, Dynamics of dendrimer-based polymer networks, J. Chem. Phys. 119 (2003) 7579.

[37] L. F. Pinto, J. Correa, M. Martin-Pastor, R. Riguera, E. Fernandez-Megia, The dynamics of dendrimers by NMR relaxation: interpretation pitfalls, J. Am. Chem. Soc. 135 (2013) 1972-1977. 
[38] L. F. Pinto, R. Riguera, E. Fernandez-Megia, Stepwise filtering of the internal layers of dendrimers by transverse-relaxation-edited NMR, J. Am. Chem. Soc. 135 (2013) 11513-11516.

[39] M. Dolgushev, A. Blumen, Dynamics of semiflexible treelike polymeric networks, J. Chem. Phys. 131 (2009) 044905.

[40] D. A. Markelov, S. G. Falkovich, I. M. Neelov, M. Y. Ilyash, V. V. Matveev, E. Lähderanta, P. Ingman, A. A. Darinskii, Molecular dynamics simulation of spin-lattice NMR relaxation in poly-l-lysine dendrimers: manifestation of the semiflexibility effect, Phys. Chem. Chem. Phys. 17 (2015) 3214-3226.

[41] D. A. Markelov, A. N. Shishkin, V. V. Matveev, A. V. Penkova, E. Lähderanta, V. I. Chizhik, Orientational mobility in dendrimer melts: Molecular dynamics simulations, Macromolecules 49 (2016) 9247.

[42] O. V. Shavykin, I. M. Neelov, A. A. Darinskii, Is the manifestation of the local dynamics in the spin-lattice nmr relaxation in dendrimers sensitive to excluded volume interactions?, Phys. Chem. Chem. Phys. 18 (2016) 2430724317.

[43] M. Dolgushev, S. Schnell, D. A. Markelov, Local NMR relaxation of dendrimers in the presence of hydrodynamic interactions, Appl. Magn. Reson. 48 (2017) 657-671.

[44] M. Dolgushev, D. A. Markelov, F. Fürstenberg, T. Guérin, Local orientational mobility in regular hyperbranched polymers, Phys. Rev. E 94 (2016) 012502 .

[45] T. Vicsek, Fractal models for diffusion controlled aggregation, J. Phys. A: Math. Gen. 16 (1983) L647.

[46] C. Jayanthi, S. Wu, J. Cocks, Real space green's function approach to vibrational dynamics of a Vicsek fractal, Phys. Rev. Lett. 69 (1992) 19551958. 
[47] C. S. Jayanthi, S. Y. Wu, Frequency spectrum of a general Vicsek fractal, Phys. Rev. B 48 (1993) 10188.

[48] C. S. Jayanthi, S. Y. Wu, Exact calculation of the dynamics of a general Vicsek fractal, Phys. Rev. B 48 (1993) 10199.

[49] C. S. Jayanthi, S. Y. Wu, Dynamics of a Vicsek fractal: The boundary effect and the interplay among the local symmetry, the self-similarity, and the structure of the fractal, Phys. Rev. B 50 (1994) 897.

[50] W. A. Schwalm, M. K. Schwalm, M. Giona, Group theoretic reduction of Laplacian dynamical problems on fractal lattices, Phys. Rev. E 55 (1997) 6741.

[51] A. Blumen, A. Jurjiu, T. Koslowski, C. von Ferber, Dynamics of Vicsek fractals, models for hyperbranched polymers, Phys. Rev. E 67 (2003) 061103.

[52] A. Blumen, C. von Ferber, A. Jurjiu, T. Koslowski, Generalized Vicsek fractals: Regular hyperbranched polymers, Macromolecules 37 (2004) 638650.

[53] T. Koslowski, A. Jurjiu, A. Blumen, Models of irregular hyperbranched polymers: Topological disorder and mechanical response, Macromol. Theory Simul. 15 (2006) 538-545.

[54] D. Zhou, Spectral analysis of Laplacians on the Vicsek set, Pacific J. Math. 241 (2009) 369-398.

[55] Z. Zhang, S. Zhou, L. Chen, M. Yin, J. Guan, The exact solution of the mean geodesic distance for Vicsek fractals, J. Phys. A: Math. Theor. 41 (2008) 485102.

[56] Z. Zhang, B. Wu, H. Zhang, S. Zhou, J. Guan, Z. Wang, Determining global mean-first-passage time of random walks on Vicsek fractals using eigenvalues of Laplacian matrices, Phys. Rev. E 81 (2010) 031118. 
[57] Y. Lin, B. Wu, Z. Z. Zhang, Determining mean first-passage time on a class of treelike regular fractals, Phys. Rev. E 82 (2010) 031140.

[58] B. Wu, Y. Lin, Z. Zhang, G. Chen, Trapping in dendrimers and regular hyperbranched polymers, J. Chem. Phys. 137 (2012) 044903.

[59] S. Constantin, R. S. Strichartz, M. Wheeler, Analysis of the Laplacian and spectral operators on the Vicsek set, Comm. Pure Appl. Analysis 10 (2011) $1-44$.

[60] A. Y. Cherny, E. M. Anitas, V. A. Osipov, A. I. Kuklin, Deterministic fractals: extracting additional information from small-angle scattering data, Phys. Rev. E 84 (2011) 036203.

[61] B. Pal, A. Chakrabarti, Staggered and extreme localization of electron states in fractal space, Phys. Rev. B 85 (2012) 214203.

[62] J. Peng, Mean trapping time for an arbitrary node on regular hyperbranched polymers, J. Stat. Mech.: Theor. Exp. (2014) P12018.

[63] J. A. Fan, et al., Fractal design concepts for stretchable electronics, Nat. Commun. 5 (2014) 3266.

[64] S. Patterson, B. Bamieh, Consensus and coherence in fractal networks, IEEE Trans. Control Netw. Syst. 1 (2014) 338-348.

[65] F. Fürstenberg, M. Dolgushev, A. Blumen, Dynamics of semiflexible regular hyperbranched polymers, J. Chem. Phys. 138 (2013) 034904.

[66] F. Fürstenberg, M. Dolgushev, A. Blumen, Exploring the applications of fractional calculus: Hierarchically built semiflexible polymers, Chaos, Solitons \& Fractals 81 (2015) 527-533.

[67] F. Fürstenberg, A. A. Gurtovenko, M. Dolgushev, A. Blumen, Molecular dynamics simulations of hyperbranched PAMAM Vicsek fractals, Macromol. Theory Simul. 24 (2015) 100-109. 
[68] N. Kulvelis, M. Dolgushev, O. Mülken, Universality at breakdown of quantum transport on complex networks, Phys. Rev. Lett. 115 (2015) 120602.

[69] M. Dolgushev, T. Guérin, A. Blumen, O. Bénichou, R. Voituriez, Contact kinetics in fractal macromolecules, Phys. Rev. Lett. 115 (2015) 208301.

[70] M. Dolgushev, H. Liu, Z. Zhang, Extended Vicsek fractals: Laplacian spectra and their applications, Phys. Rev. E 94 (2016) 052501.

[71] E. G. C. P. van Veen, S. Yuan, M. I. Katsnelson, M. Polini, A. Tomadin, Quantum transport in sierpinski carpets, Phys. Rev. B 93 (2016) 115428.

[72] O. Mülken, S. Heinzelmann, M. Dolgushev, Information dimension of stochastic processes on networks: Relating entropy production to spectral properties, J. Stat. Phys. 167 (2017) 1233-1243.

[73] M. Nikbakht, Radiative heat transfer in fractal structures, Phys. Rev. B 96 (2017) 125436.

[74] A. Jurjiu, T. L. Biter, F. Turcu, Relaxation dynamics of a multihierarchical polymer network, J. Chem. Phys. 146 (2017) 034902.

[75] A. S. Balankin, Mapping physical problems on fractals onto boundary value problems within continuum framework, Phys. Lett. A 382 (2018) 141-146.

[76] A. Jurjiu, R. Dockhorn, O. Mironova, J.-U. Sommer, Two universality classes for random hyperbranched polymers, Soft Matter 10 (2014) 49354946.

[77] N. Madras, E. J. J. van Rensburg, Monte carlo study of the $\theta$-point for collapsing trees, J. Stat. Phys. 86 (1997) 1-36.

[78] H.-K. Janssen, O. Stenull, Collapse transition of randomly branched polymers: Renormalized field theory, Phys. Rev. E 83 (2011) 051126.

[79] A. Y. Grosberg, Annealed lattice animal model and flory theory for the melt of non-concatenated rings: towards the physics of crumpling, Soft Matter 10 (2014) 560-565. 
[80] A. Rosa, R. Everaers, Computer simulations of melts of randomly branching polymers, J. Chem. Phys. 145 (2016) 164906.

[81] S. Alexander, R. Orbach, Density of states on fractals: fractons, J. Phys. Lett. 43 (1982) 625-631.

[82] M. L. Mansfield, W. H. Stockmayer, Unperturbed dimensions of wormlike stars, Macromolecules 13 (1980) 1713-1715.

[83] T. Khazanovich, Theory of nuclear magnetic relaxation in liquid-phase polymers, Polym. Sci. U.S.S.R. 4 (1963) 727-736.

[84] A. Perico, M. Guenza, Viscoelastic relaxation of segment orientation in dilute polymer solutions, J. Chem. Phys. 83 (1985) 3103-3109.

[85] A. Abragam, The principles of nuclear magnetism, Oxford university press, 1961.

[86] V. I. Chizhik, Y. S. Chernyshev, A. V. Donets, V. V. Frolov, A. V. Komolkin, M. G. Shelyapina, Magnetic resonance and its applications, Springer, 2014.

[87] N. F. Fatkullin, T. Körber, E. A. Rössler, Signature of reptation in the longtime behavior of the deuteron NMR free induction decay in high molecular mass polymer melts, Polymerdoi:10.1016/j.polymer.2018.03.050

[88] A. A. Gurtovenko, Y. Y. Gotlib, Intra-and interchain relaxation processes in meshlike polymer networks, Macromolecules 31 (1998) 5756-5770.

[89] F. Mohamed, M. Hofmann, B. Pötzschner, N. Fatkullin, E. A. Rössler, Dynamics of PPI dendrimers: A study by dielectric and 2H NMR spectroscopy and by field-cycling $1 \mathrm{H}$ NMR relaxometry, Macromolecules 48 (2015) 3294-3302. 


\section{Highlights}

- The spectral density $[J(\omega)]=\omega J(\omega)$ involved in the NMR relaxation functions has for fractal macromolecules very strong features: for the peripheral segments it is characterized by a single time (like for dendrimers); for the inner segments one observes a significant broadening of $[J(\omega)]$; the segments located deeply inside the macromolecule do not feel its size anymore (like in a network).

- The inclusion of local stiffness is fundamental for the NMR relaxation of branched macromolecules. In the absence of semiflexibility the spectral density has (spuriously) the maximum located at the same frequency, independently of the segment's location. 


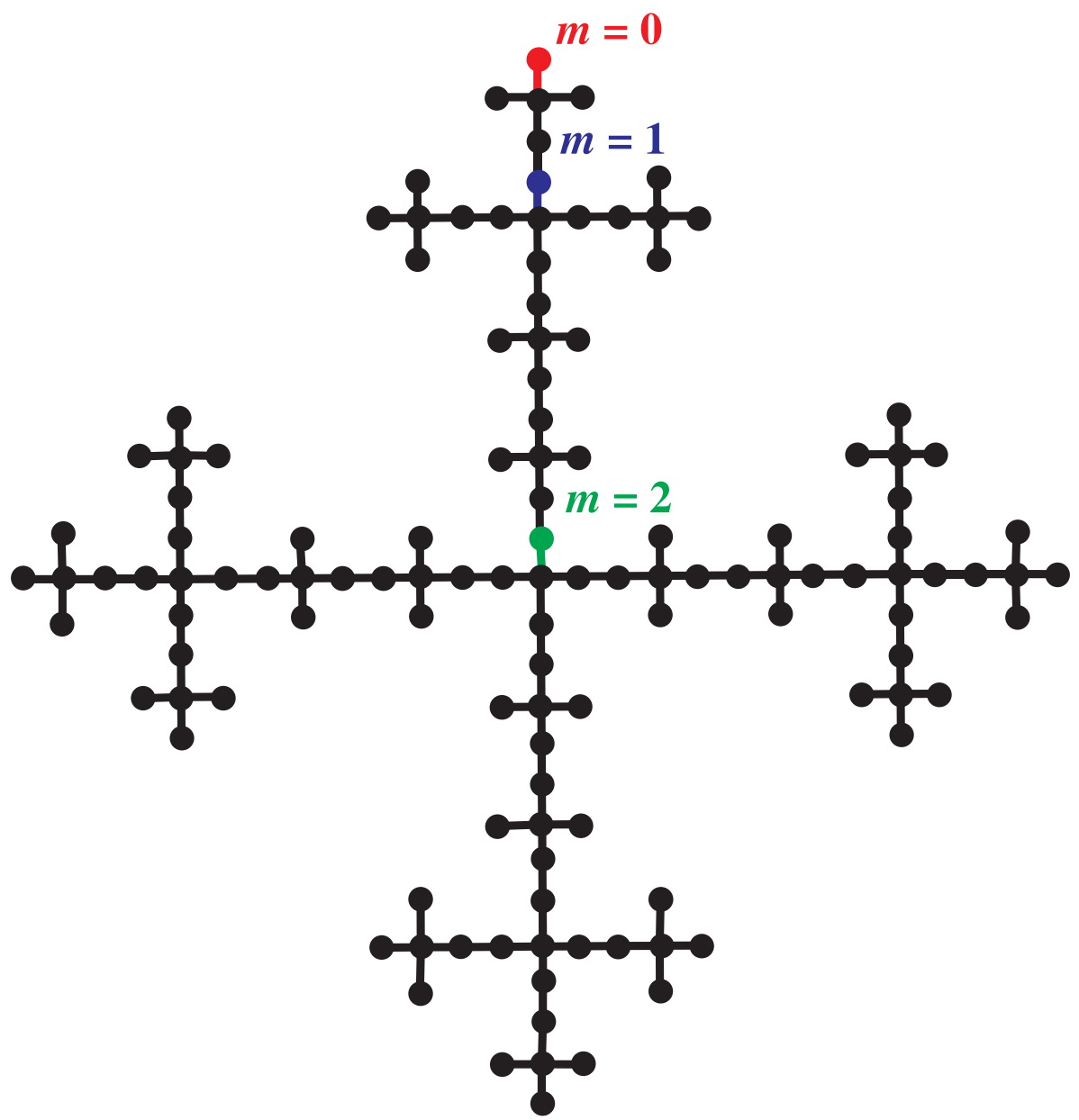

Figure 1: Sketch of a Vicsek fractal (VF) of generation $G=3$ and functionality of branched units $F=4$. Exemplarily the core segments of different (sub)branches are indicated: $m=0$ (red), $m=1$ (blue), $m=2$ (green). Note that the segments are counted from the periphery. 

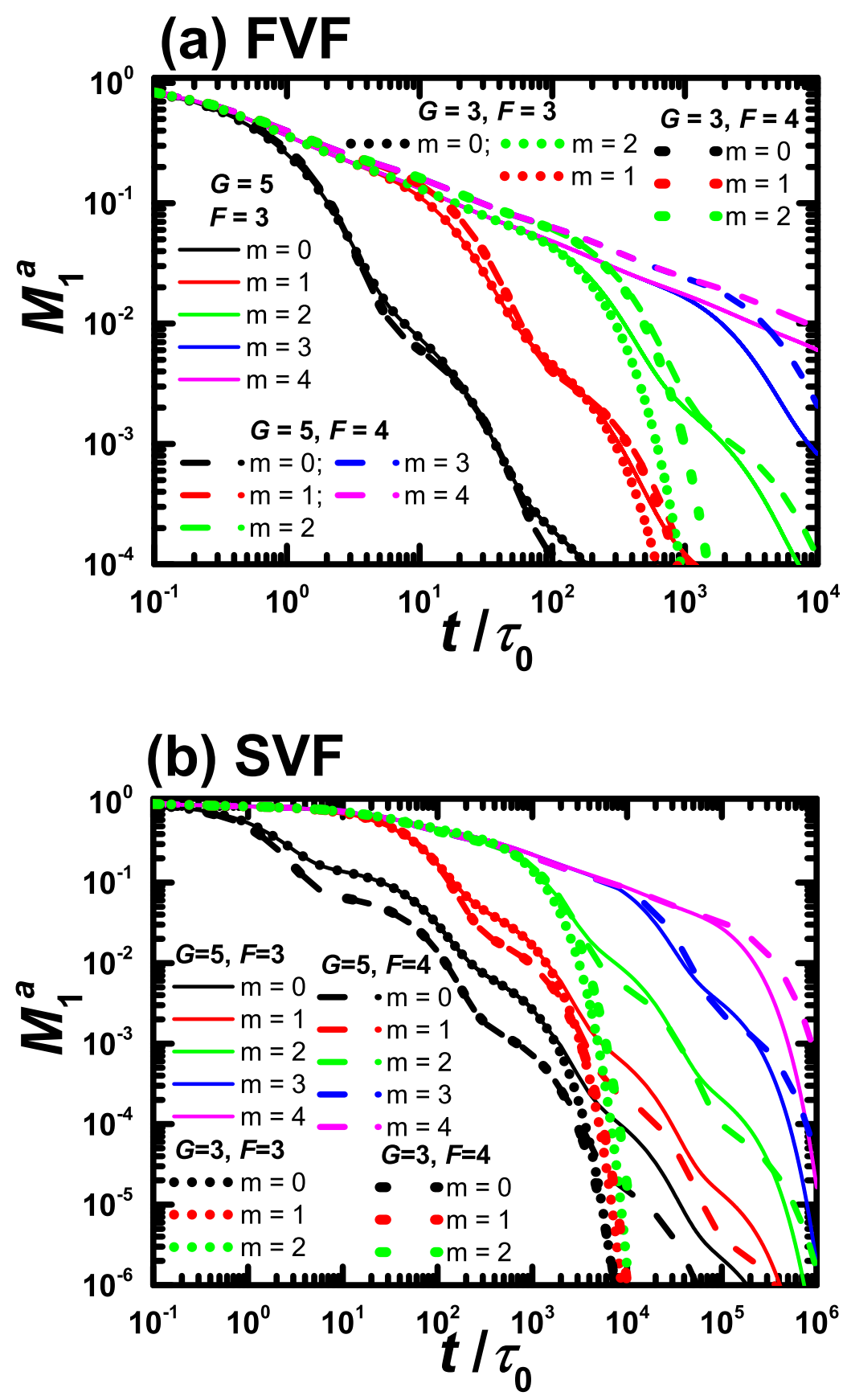

Figure 2: Bond-bond autocorrelation function $M_{1}^{a}(t)$ for segments of flexible VF [FVF, part (a)] and semiflexible VF [SVF, part (b)] for various parameters $m, G, F$, indicated in the legends. 

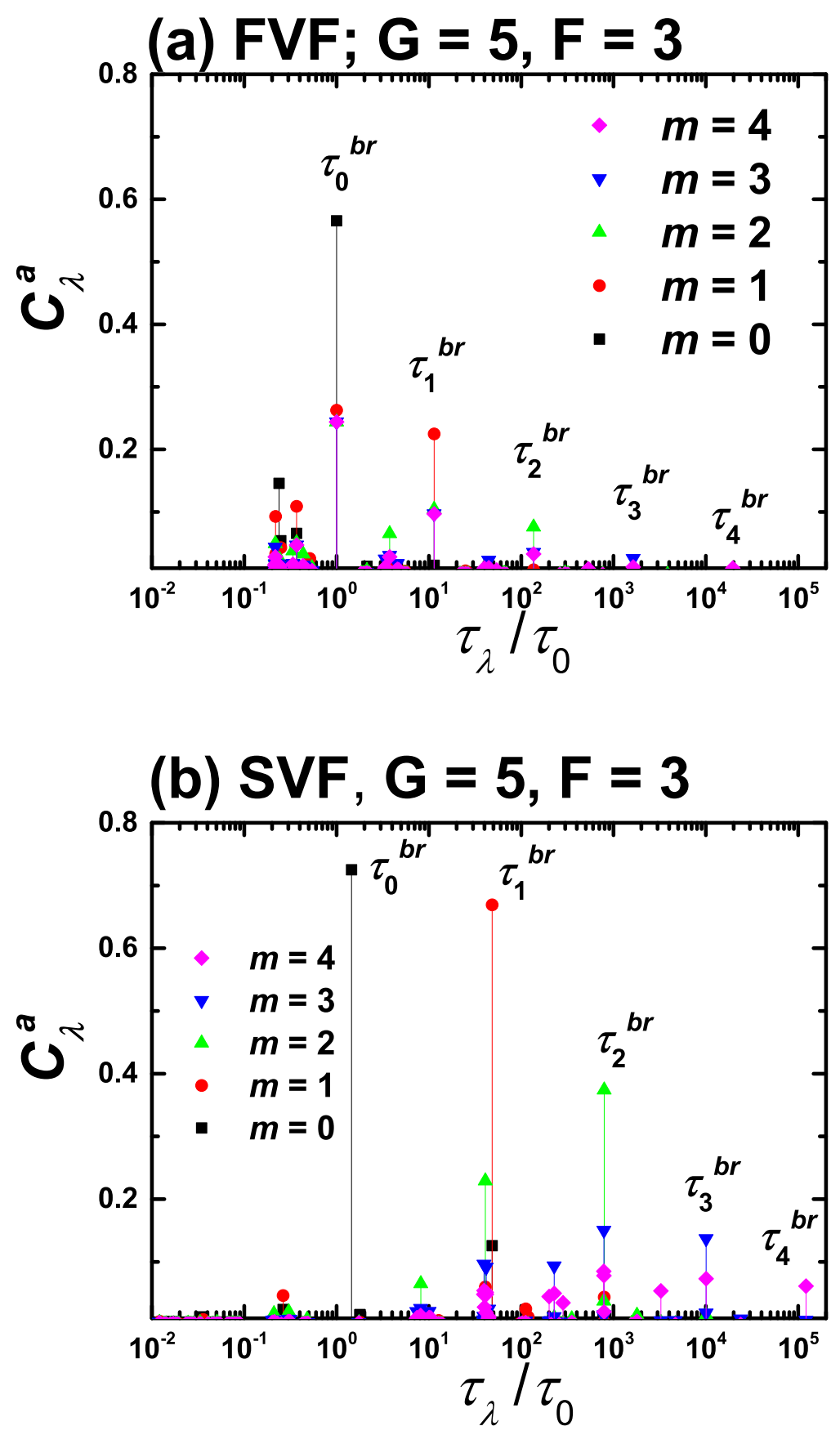

Figure 3: Contributions $C_{\lambda}$ of the relaxation times $\tau_{\lambda}$ to the decay of $M_{1}^{a}(t)$ for FVF (a) and SVF (b) of functionality $F=3$ and generation $G=5$. 


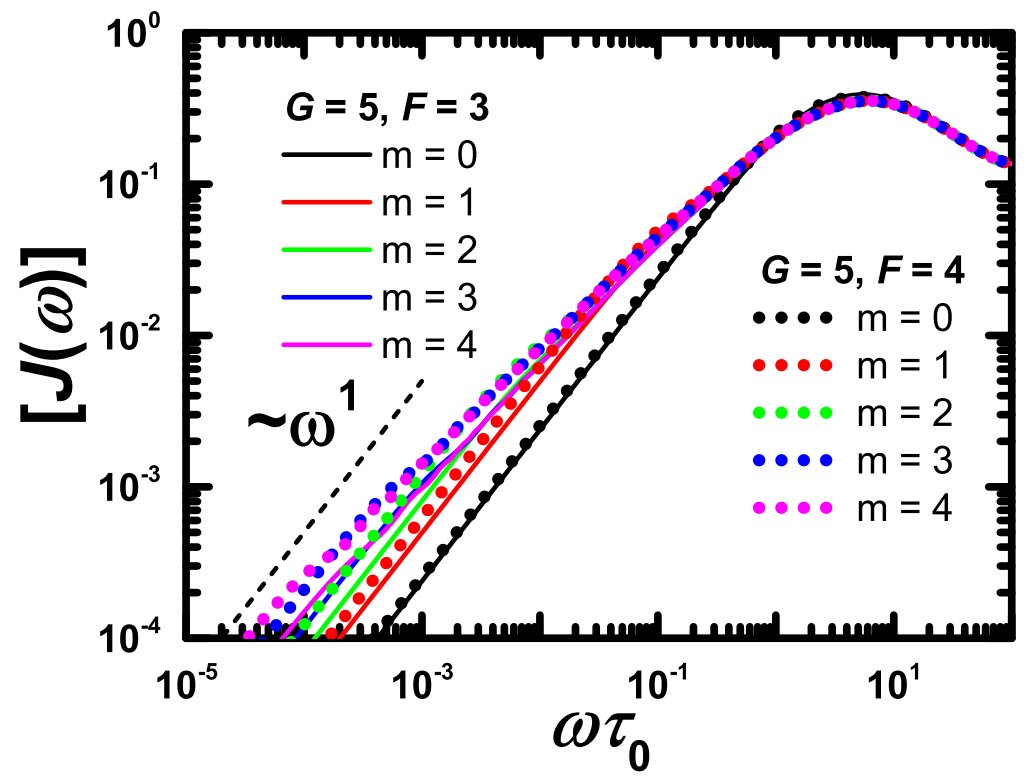

Figure 4: Reduced spectral density $[J(\omega)]$ for FVF of generation $G=5$ and $F=3$ and 4 . 

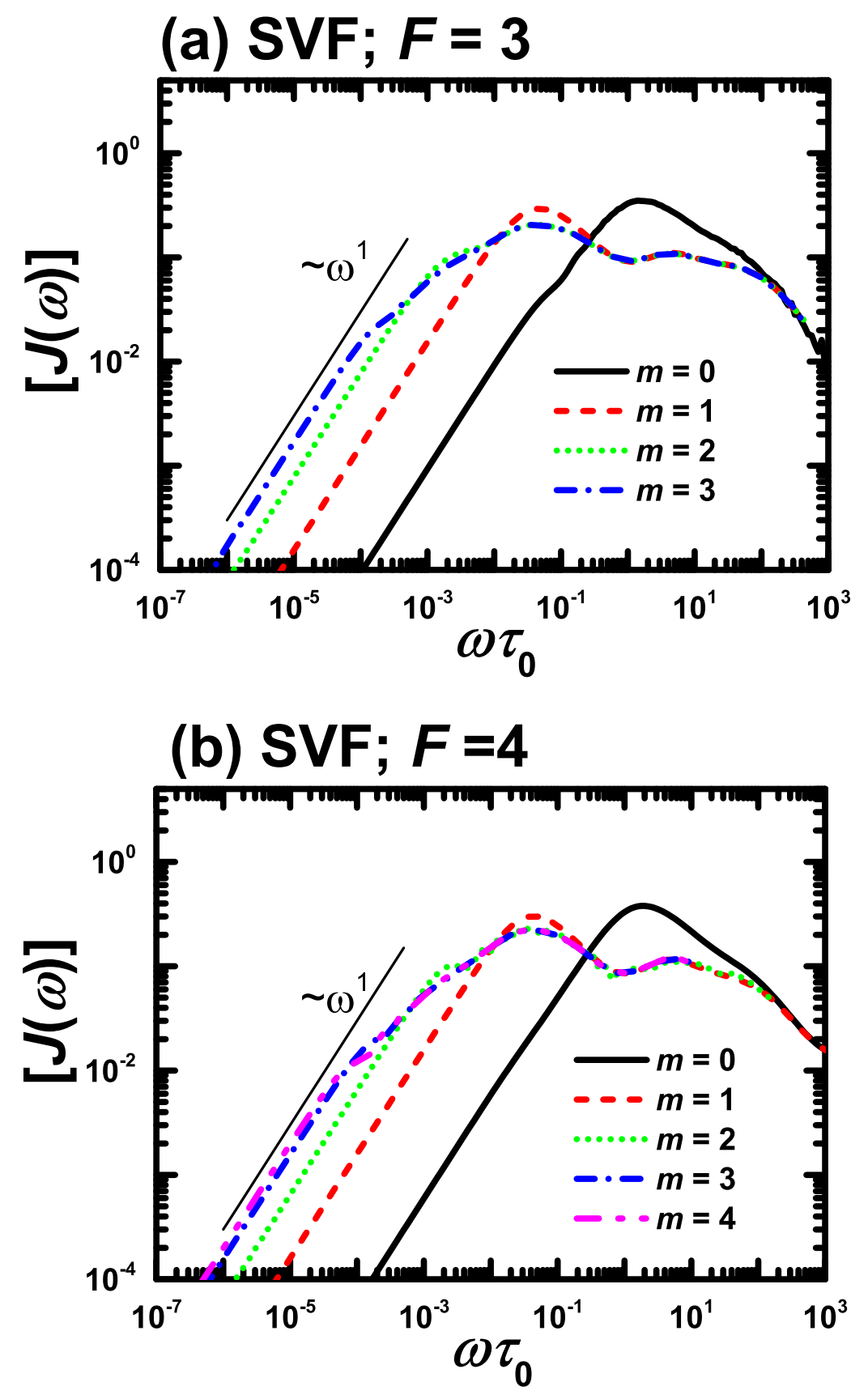

Figure 5: Reduced spectral density $[J(\omega)]$ for SVF of functionality $F=3$ (a) and $F=4$ (b) at generation $G=5$. 


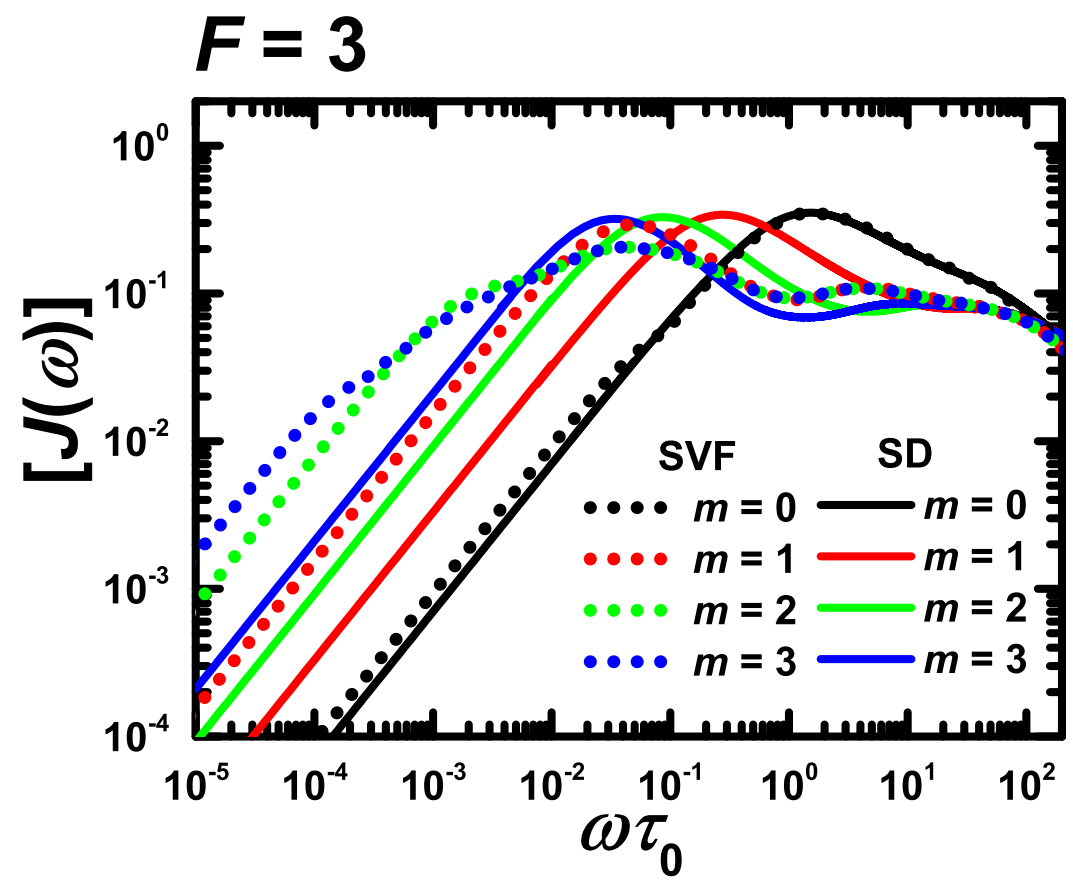

Figure 6: Comparison of the reduced spectral densities $[J(\omega)]$ for SVF and semiflexible dendrimer (SD), both of functionality $F=3$, generation $G=5$, and stiffness parameter $q_{\mathrm{br}}=0.45$. The data for SD are taken from Ref. [4]. 
Graphical abstract
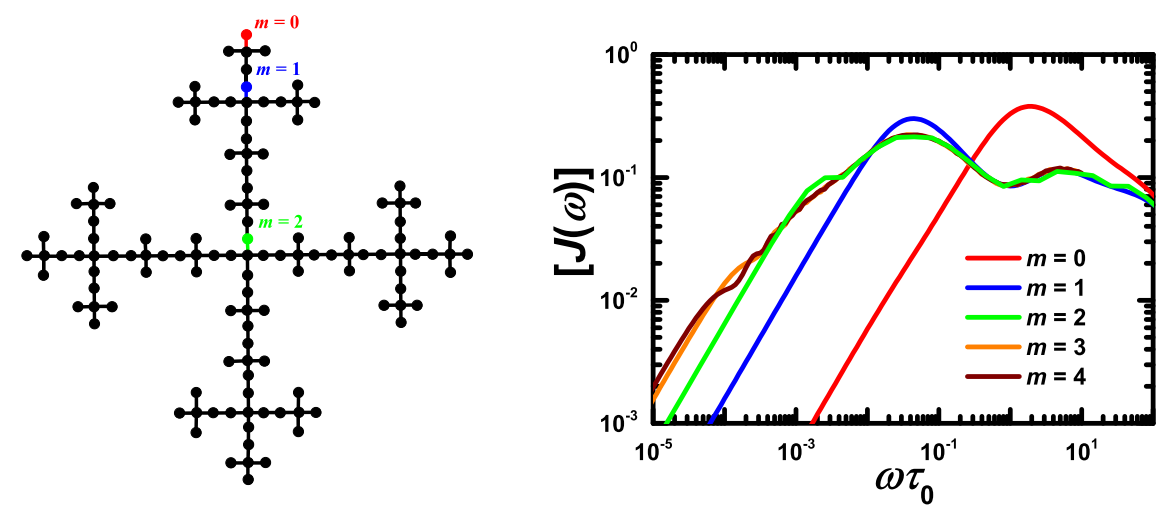